\title{
Review on research of water saving for natural ventilation cooling tower
}

\author{
Shuyuan $\operatorname{Ran}^{1}$ \\ ${ }^{1}$ China Energy Engineering Group Jiangsu Power Design Institute Co., Ltd, Jiangning District, Nanjing City, Jiangsu Province, 210012, \\ China
}

\begin{abstract}
Thermal power plant is one of the eight high water consumption industries in China, and circulating cooling water is the largest water consumption project. Under the increasingly severe situation of water resources in China, measures must be taken to reduce the evaporation loss, wind blowing loss and pollution discharge loss of natural ventilation cooling tower. Based on the comprehensive summary of water-saving technology of natural ventilation cooling tower, we compared the advantages and disadvantages of various technologies to provide a basis for water-saving transformation of cooling tower.
\end{abstract}

\section{Introduction}

As thermal power plants require a large amount of water for cooling, the installed capacity and the cooling water demand increase with the development of China's electric power industry. Since the 1960s, the direct current water supply mode has been gradually eliminated due to large water consumption and the generation of heat pollution, and it is replaced by the cooling tower with secondary water supply cycle or the cooling tower with air cooling mode. Moreover, the design, construction and operation of the cooling tower have achieved rich experience and considerable development owing to the development of China's electric power industry in recent years.

Natural draft cooling tower, also known as hyperbolic cooling tower, air flow more smoothly, which is advantageous in ventilation, and occupies a dominant position in China's electric power system. The cooling tower uses the temperature and pressure difference generated by the hot air in the tower to make the ventilation tube produce enough pumping force, which enables cold air to continuously flow into the tower and has sufficient heat exchange with the hot water to cool the hot water. Natural draft cooling towers are divided into wet cooling tower and air cooling tower. The air cooling tower completes the heat transfer between the being cooled medium (circulating cooling water) and the cooling medium (air) by arranging and supporting radiators and related pipes.

The water of natural draft cooling tower is mainly consumed in three aspects: evaporation loss, wind blowing loss and sewage loss. Among which, the evaporation loss is about $1.2-1.6 \%$ of the circulating water, accounting for $30-55 \%$ of the total water consumption of the power plant, and it is the largest index of the water consumption of power plant. With the development of industry and agriculture and the improvement of people's living standards, the contradiction between supply and demand of fresh water in our country is becoming increasingly acute and the shortage of water resources have seriously restricted the speed and development of China's economic construction, especially in the coastal economically developed cities. Therefore, it is necessary to take effective actions to realize water saving of cooling tower.

\section{Current situation of water saving technology}

\subsection{Technology of reducing evaporation loss}

\subsubsection{Water saving technology of atomization cooling to reduce evaporation loss}

In order to reduce the evaporation loss, the atomization cooling water-saving technology can be adopted. This technology is fulfilled by installing a condensing ejector is installed in the middle of the cooling tower, and cold water is used as the condensing agent. Before the heat exchange between air and water, the temperature of circulating cooling water is reduced, so that the amount of saturated wet air formed above the cooling tower is less. Low temperature water is atomized into small water droplets and evenly sprayed on the cross section of the cooling tower. The small water droplets whose temperature is lower than that of the hot and humid air absorb the latent heat released by the water vapor in the saturated wet air layer in the tower, condense the water vapor and reduce the evaporation loss. However, after the installation of atomization cooling device, the ventilation resistance of the cooling tower will increase and thus the ventilation volume will decrease. However, 
the ventilation volume needs to be increased in order to ensure the heat transfer effect of condensate water and air, Therefore, after installing the atomization cooling device, induced draft fans need to be installed to increase the ventilation volume of the cooling-tower.

\subsubsection{High voltage electrostatic deposition technology to reduce evaporation loss}

High voltage electrostatic precipitation technology applies the theory of electrostatic precipitation to recover $5 \sim 10 \mu \mathrm{m}$ water droplets from the water vapour from the wet cooling tower. When the micro water particles evaporated from the cooling tower of thermal power plant pass through the high voltage electric field, they are charged and become charged water particles, and they will move to the opposite electrode and finally reach the opposite electrode under the action of electric field. When the charged micro water particles reach a certain concentration at the electrode, a thin film of water is formed until it begins to flow, so that the water on the electrode can be recovered by containers or pipes to reduce evaporation loss [1]. After the installation of high-voltage electrostatic precipitator, the water consumption can be reduced effectively. Some studies reveal that the water consumption rate can be reduced from $65 \%$ to $43 \%$, and a quantitative test shows that the water collection rate of high-voltage electrostatic precipitator is about $41 \%$ for a $200 \mathrm{MW}$ unit. Additionally, the device can also reduce the tower height of the new natural ventilation wet cooling tower, reduce the airflow resistance of the natural ventilation wet cooling tower, reduce the temperature of the circulating cooling water, and improve the quality of the circulating cooling water [2]. Although this device can recover the small droplets within a certain range visible in the tower, it still cannot recover most of the invisible saturated water vapour existing in the saturated wet air in the tower. Moreover, the device consumes a lot of electric energy, the operation cost is considerable, and the Water-saving Benefit is not enough to offset the investment cost, so the high-voltage electrostatic device cannot be applied engineering practice.

\subsubsection{Reduce evaporation loss by cooling and condensing water}

Cooling the saturated wet air above the water collector of the cooling tower can condense the water vapour in the air and recover the condensed water to reduce the evaporation loss. And this is fulfilled by using heat pipe technology to condense saturated wet air, and installing injection device to add condensation nodules to the air, so as to accelerate the condensation of water vapour in the air, and using water collecting net made of polyethylene to recover the condensed water. Theoretical analysis shows that $11 \%$ of evaporation loss of cooling tower can be recovered by cooling saturated wet air at $2^{\circ}$ $\mathrm{C}[3,4]$. However, due to the high cost of heat pipe, the installation and layout of its condensate device is also relatively complex, there is limitation on the practical application and promotion of this technology [5].

\subsection{Technology of reducing wind consumption}

\subsubsection{Using water remover to reduce wind consumption}

When the water remover is not installed, the wind loss accounts for about $0.5 \%$ of the circulating water. And after the water remover is installed, the wind loss is reduced to about $0.1 \%$ of the circulating water. If taking a $20 \mathrm{MW}$ motor unit into consideration, the wind loss can be reduced by about $100 \mathrm{~m}^{3} / \mathrm{h} \times 7000 \mathrm{~h}=70 \times 10^{4} \mathrm{~m}^{3} / \mathrm{a}$ every year, and the investment of the water remover can be recovered in about 4 years. Therefore, the installation of water separator can effectively reduce the wind loss. In Technical specification for design of thermal power plant (SDJ1-84) it has been clearly regulated that "new cooling tower shall be equipped with water remover". The water eliminator can almost remove floating droplets with water particle size larger than $100 \mu \mathrm{m}$. In addition to the traditional corrugated plate or character "八" type water remover, various new and efficient water removers have been developed and applied. For example, the highefficiency cyclone water remover diverts the wet air into a rotating flow, and uses centrifugal force to throw the condensed water to the tower wall for recovery. Meanwhile, it enables the contact time between the wet air and the wall increase to improve the condensation efficiency; the high-voltage electrostatic water remover uses the electrostatic principle to make the wet air obtain electric charge, polarize, and be attracted to the electrode, which achieve the purpose of water saving. Experiment reveals that under the same conditions, the water collection efficiency is increased by $10-20 \%$, but the pressure drop of high efficiency cyclone collector is larger than that of corrugated plate collector [6].

\subsubsection{Adopting thin and high tower type design to reduce wind consumption}

The ratio of tower height to zero-meter diameter (height diameter ratio) of conventional natural draft cooling tower is generally $1.2 \sim 1.4$, with low tower height, large zero-meter diameter and a large floor area, it is the most commonly used cooling tower type in thermal power plant. However, the height diameter ratio of thin and high natural draft cooling tower is generally more than 1.5. On the premise of ensuring the cooling effect, the zero-meter diameter is relatively small, the floor area is less, the tower body is higher, and the cooling capacity of air extraction is stronger. The results show that the highefficiency water collector can reduce the wind loss rate to $0.001 \%$. Compared with the conventional cooling tower, the high-efficiency water-saving thin high cooling tower can save $10.2 \times 104 \mathrm{~m} 3$ of water every year, and the water-saving effect is remarkable. In addition, the thin and high natural draft cooling tower also has the characteristics of power saving, noise reduction, 
antifreeze, flexible operation and small maintenance workload [7].

\subsection{Technology of reducing pollution loss--- increasing concentration multiple}

Concentration multiple refers to the ratio of salt concentration of circulating cooling water to that of make-up water. And the higher concentration multiple means the higher reuse rate of water, the less sewage discharge, and the less make-up water. The main measure to increase the concentration multiple is to add chemical agents to treat the circulating water, such as organic scale inhibitor, corrosion inhibitor, bactericide and algaecide, which can increase the concentration multiple to 4-8. Niagara Falls thermal power plant in the United States adopts the conventional phosphorus series formula and operates well under the condition of concentration multiple of 10 , while the concentration multiple of AEC treatment scheme can reach 19-22 [3]. There is still a long way to go to improve the concentration ratio of circulating water in China and this can be achieved by continuously developing new reagents and applying new materials and technologies in the condenser heat exchange tube.

In recent years, non-chemical methods have also been included in the research field, such as magnetization treatment, electric field treatment and ultrasonic treatment technology. However, if the concentration ratio is too large, the water-saving effect will reduce, and the concentration ratio will continuously increase, resulting in increasingly strict requirements on fungicides, water treatment agents, system facilities and management, and increase the phenomenon of corrosion, scaling, mud deposition, lead to the growth of micro-organisms. For the circulating water with increased concentration ratio, on one hand, enhanced heat transfer technology can be applied to allow the existence of larger fouling thermal resistance on the premise of ensuring the total heat transfer effect of the condenser unchanged, and "water saving" and "energy saving" can be considered comprehensively; on the other hand, new heat exchange materials and heat exchange technology can be developed to the condenser heat exchange tube to stabilize the water quality and optimize the operation mode. In order to select concentration multiple scientifically and reasonably, He Jing et al. used multiattribute decision-making method (TOPSIS) to optimize the concentration multiple, and provide a basis for water saving and consumption reduction [8].

\subsection{Other water saving measures}

\subsubsection{Using air cooling technology to save water}

In conventional wet cooling towers, the circulating water is in direct contact with the air in the way of "rain" for heat exchange, and the tower is in the "wet" state, its cooling system is called the wet cooling system. However, in the air cooling technology, the circulating water is in indirect contact with the air through the radiator for heat exchange, and the tower is in the "dry" state, and its cooling system is called the dry cooling system. Air cooling technology can avoid the loss of evaporation, wind and sewage of wet cooling tower, which maximize the purpose of water saving in power plant. However, because air cooling technology relies on contact heat transfer, the cooling efficiency is low and the cooling water temperature is high, resulting in the reduction of unit efficiency and the increase of power consumption. In addition, the metal tube of air cooling technology increases the cost of cooling tower. Therefore, in recent years, traditional wet cooling towers have been widely replaced by air-cooled units, especially in the "rich coal and water shortage" areas, such as Shanxi, Shaanxi, Inner Mongolia and so on.

\subsubsection{Reuse of sewage as make-up water}

The reuse of sewage as supplementary water has achieved the double benefits of saving water resources and reducing environmental pollution, which is widely used at home and abroad. For example, American power plants use secondary effluent from sewage treatment plants, various kinds of wastewater from power plants, roof rainwater, and treated wastewater from cooling towers as supplementary water for circulating cooling water. In our country, various kinds of sewage are used as the supplementary water of circulating water, such as secondary treated urban sewage, domestic sewage, fish pond drainage and domestic sewage after treatment by biological aerated filter, coagulation flotation, filtration, disinfection and other processes. However, the wastewater reuse technology does not reduce the water consumption of the cooling tower in essence, but only save the fresh water as the make-up water of the cooling tower, and takes the reused wastewater as the substitute of the make-up water.

\section{Conclusion}

At present, the relatively mature and the practical applied water-saving technology of natural draft cooling tower is the installation of water collector, increasing of concentration ratio, and the use of air cooling technology. It is still the main direction of water-saving transformation of cooling tower in the future to study the use of high-efficiency and low resistance water collector to reduce the wind blowing loss, develop new chemicals and new formulations, apply new materials and new technologies to the heat exchange tube of condenser, improve the concentration ratio and reduce the sewage loss. However, there are still many problems remain to be solved through the cooling technology of saturated wet air.

\section{References}

1. Kanghong Ning, Tiexin Hou, Naiqiu Shu, Lingwei Zheng. (2003) Method of reducing evaporation water consumption based on high voltage electric 
field. Electric power construction. 24(5): 59-60, 639 (in Chinese).

2. Tianzheng Wang, Peizhang Wang. (1995) Water saving technology of installing high voltage electrostatic suction and floating water collector in natural draft cooling tower. China Electric Power. 9: 65-67, 74 (in Chinese).

3. Duncai Huo, Chaoran Jin, Lu Gan. (2017) Water loss analysis and water saving technology of counter current wet cooling tower. Chemical engineering management. 8:161 (in Chinese).

4. Fang Li, Jinggang Wang, Jinrong Liu. (2005) Theoretical analysis of water saving in cooling tower with heat pipe technology. Proceedings of the Third Symposium on new technology of refrigeration and air conditioning, Hangzhou.: 446-450 (in Chinese).

5. Junfeng Li, Yanbin Wu, Guoquan Song, Guangxue Yan, Jinling Cui. (2017) Discussion on water saving method of counter current cooling tower. Fine and special chemicals. 25 (3):20-23 (in Chinese).

6. Ruqing Liu, Fengzhong Sun, Youliang Chen, Dan Zhou. (2007) Discussion on water saving technology of counter current wet cooling tower. Power station system engineering. 23 (6): 47-51 (in Chinese).

7. Jun Zhou. (2016) Application of high efficiency and water saving thin and tall natural draft cooling tower in gas turbine power plant. Power generation and air conditioning. 172(37): 25-28 (in Chinese).

8. Jing He, Ming Zhao, Farong Shen, Ping He. (2013) Optimization of concentration ratio and water saving of cooling tower based on TOPSIS. Industrial heating. 42(6):49-52 (in Chinese). 